\title{
Time for large randomised trials of vitamin D for women with gestational diabetes mellitus to improve perinatal health outcomes
}

\author{
Heather H. Burris • Carlos A. Camargo Jr
}

Received: 9 June 2014 / Accepted: 17 June 2014 / Published online: 4 July 2014

(C) Springer-Verlag Berlin Heidelberg 2014

\begin{abstract}
Gestational diabetes mellitus (GDM) affects $7-14 \%$ of pregnancies in the USA and its incidence is rising. Several small trials have demonstrated improved insulin sensitivity and glucose tolerance with vitamin D supplementation among women with GDM. In this issue of Diabetologia (DOI: 10.1007/s00125-014-3293-x), Asemi and colleagues report that co-supplementation of vitamin $\mathrm{D}$ and calcium among women with diet-controlled GDM improves several biomarkers of metabolic status, including insulin, glucose and cholesterol. Whether such improvements in biomarkers would translate into clinically meaningful reductions in the harmful sequelae of GDM remains unknown. The promising results from Asemi and colleagues justify investment in large randomised, double-blind, placebo-controlled trials of vitamin D (with or without calcium) for women with GDM. Such trials should be powered for perinatal outcomes such as Caesarean section for macrosomia, postnatal hypoglycaemia and respiratory distress; ideally, the trials would include long-term followup of mothers and infants for later metabolic consequences of GDM.
\end{abstract}

Keywords GDM · Gestational diabetes · Pregnancy · Vitamin D

H. H. Burris $(\bowtie)$

Department of Neonatology, Beth Israel Deaconess Medical Center, 330 Brookline Ave, RO 318, Boston, MA 02215, USA

e-mail: heburris@bidmc.harvard.edu

H. H. Burris

Division of Newborn Medicine, Department of Pediatrics at Boston Children's Hospital, Harvard Medical School, Boston, MA, USA

C. A. Camargo Jr

Department of Emergency Medicine, Massachusetts General

Hospital, Harvard Medical School, Boston, MA, USA

\author{
Abbreviations \\ 25(OH)D 25-Hydroxyvitamin D \\ ACOG American College of Obstetricians and \\ Gynecologists \\ GDM Gestational diabetes mellitus \\ NICU Neonatal intensive care unit \\ RCT Randomised controlled trial
}

Gestational diabetes mellitus (GDM) affects $7-14 \%$ of pregnancies in the USA $[1,2]$ and its incidence is increasing worldwide [3]. While increased screening and more sensitive protocols may be partially responsible [4], there is little doubt that the rise in GDM is tightly linked to the obesity epidemic and shifting demographics among pregnant women. Compared with prior generations, pregnant American women are heavier, older and more likely to be from a racial/ethnic group (Asian, Hispanic, African-American) at higher risk of GDM [5]. GDM leads to several maternal and neonatal morbidities that can have both acute and lifelong consequences. For example, mothers with GDM are more likely to undergo Caesarean section [6], thereby incurring surgical risks, and they are more likely to develop type 2 diabetes later in life [7]. Likewise, infants born to women with GDM are more likely to have congenital anomalies, macrosomia leading to birth trauma, hypoglycaemia, respiratory distress, polycythaemia and jaundice [4]. All of these complications are associated with prolonged hospital stays in the newborn period often requiring admission to the neonatal intensive care unit (NICU), but infants born to women with GDM may also suffer life-long negative health effects. For example, infants born to mothers with GDM are at risk of developing abnormal glucose tolerance, obesity and the metabolic syndrome - factors that contribute to cardiovascular disease during adulthood [3].

Investigators have identified several risk factors for GDM, including advanced maternal age, obesity, family history of 
diabetes and specific racial/ethnic backgrounds [8]. Nevertheless, the mechanism(s) for how these factors lead to GDM remain poorly understood. Vitamin D deficiency has become an active area of investigation among GDM researchers because it may provide a unifying mechanism to explain how several of these risk factors affect GDM risk [8]. For example, obesity is a shared risk factor for both vitamin D deficiency [9] and GDM [10]. Moreover, epidemiological studies have demonstrated a link between vitamin D deficiency and a higher risk of type 2 diabetes mellitus [11]. These observations have led investigators to focus on vitamin D deficiency as a potential therapeutic target to prevent GDM and to improve glycaemic control among women with GDM. Indeed, several observational studies have demonstrated links between low 25-hydroxyvitamin D [25(OH)D] levels (the best available marker of vitamin D status) and higher risk of GDM [12]. Even null studies have shown inverse associations between $25(\mathrm{OH}) \mathrm{D}$ and blood glucose measurements $[13,14]$. Rat models also support a role for vitamin D supplementation to improve glucose tolerance and insulin secretion $[15,16]$. More importantly, small randomised controlled trials (RCTs) of vitamin D supplementation in pregnant women have shown some promising results with respect to GDM and glycaemic control $[17,18]$.

In the current issue, Asemi and colleagues report the results of an RCT that examined the role of vitamin D (in combination with calcium) vs placebo among pregnant women with GDM [19]. Briefly, the investigators randomised 56 Iranian women with diet-controlled GDM to receive $1,000 \mathrm{mg}$ of calcium per day and a 50,000 IU vitamin $\mathrm{D}_{3}$ pearl twice over a 6 week period (date of enrolment and then 21 days later). The investigators studied several metabolic outcomes at baseline and again 6 weeks after the intervention, including fasting glucose, insulin sensitivity measures, cholesterol and inflammatory markers. Fifty-one participants completed the study ( $n=25$ in the intervention group and $n=26$ in the placebo group). The investigators report several favourable changes in metabolic markers in those who received calcium-vitamin D co-supplementation vs the placebo group. These changes included reductions from baseline in fasting plasma glucose, HOMA-IR and LDL-cholesterol. Additionally they observed favourable increases from baseline of both HDL-cholesterol and total glutathione. Several other biomarkers did not change, including C-reactive protein. This small RCT was well conducted in a relatively homogenous group of women with diet-controlled GDM. The participants were blinded to their assigned group, but the midwife administering the supplements was not. This is unlikely to be a major source of bias, but, potentially, if the midwife somehow unblinded the participants, then other behavioural changes associated with the optimism being in the 'treatment arm' (such as a motivation to exercise) may have contributed to the group differences. Another modest limitation is that the trial was limited to women who did not require insulin, which raises questions about generalisability of the findings to all women with GDM. Nevertheless, the results remain highly relevant, since approximately $90 \%$ of all women with GDM are managed with diet alone [20].

While Asemi and colleagues have demonstrated improvements in biomarkers of metabolic health with their 6 week intervention among women with GDM [19], they do not address actual health outcomes. Thus, the critical question as to the clinical utility of vitamin D supplementation in women with GDM is not addressed by this study, nor by its predecessors - namely, would vitamin D supplementation (with or without calcium) improve maternal and infant health outcomes? For example, would the observed improvements in glycaemic control translate into fewer Caesarean sections for macrosomia? Would fewer babies have postnatal hypoglycaemia? Would there be a lower frequency of respiratory distress syndrome among infants of diabetic mothers? Would there be a reduction in the length of NICU stays, or a reduction in NICU admissions overall? Perhaps even more importantly, would there be improvements in the long-term metabolic status and health outcomes of treated women and their infants? Would these women have a lower risk of developing type 2 diabetes? Would they be less likely to develop the metabolic syndrome and therefore less likely to suffer from stroke and myocardial infarction? Would their children be less likely to develop obesity and its associated morbidities?

All of these questions require answers and the elegant RCT by Asemi et al strongly encourages the design and implementation of new trials that can answer these questions. While a large RCT of vitamin D supplementation for women at risk of GDM during pregnancy (DALI) is currently under way, women with GDM are excluded [21]. Analogous RCTs of vitamin $\mathrm{D}$ for women with GDM are needed, with adequate statistical power to address relevant perinatal outcomes such as Caesarean section for macrosomia, postnatal hypoglycaemia and neonatal respiratory distress. If shown to be clinically effective, it is likely that vitamin D supplements (with or without calcium) would be very cost-effective in the subset of pregnant women with GDM. Ideally, formal cost-effectiveness analyses should be performed alongside all of these RCTs to help to motivate a meaningful change in practice.

Prior to the completion of these future trials, clinicians continue to face decisions about what to do about the vitamin D status of pregnant women with GDM. The current stance of the American College of Obstetricians and Gynecologists (ACOG) on vitamin D status screening states that there is insufficient evidence to screen all women during pregnancy for vitamin D deficiency, but that vitamin D screening with serum $25(\mathrm{OH}) \mathrm{D}$ levels may be considered for women at high risk of vitamin D deficiency [22]. Included in the ACOG description of 'high-risk' women are those who live in 
countries with limited daylight for several months of the year, or wear clothing to protect against exposure to the sun and cold, and ethnic minorities, particularly women with darker skin. At the moment, the observational literature would suggest that, on average, women with GDM have lower 25(OH)D levels than their non-diabetic pregnant counterparts [12]. Thus, it seems reasonable to screen women with GDM for vitamin D deficiency and to ensure that these women are getting adequate supplementation to achieve sufficiency. However, we should note that an optimal serum 25(OH)D level in pregnancy has not yet been established. ACOG suggests two cut-off points: $50 \mathrm{nmol} / 1 \mathrm{l}(20 \mathrm{ng} / \mathrm{ml})$ or $80 \mathrm{nmol} / 1$ $(32 \mathrm{ng} / \mathrm{ml})$. They then suggest that if mothers have 25(OH)D levels in the deficient range, that 1,000-2,000 IU of vitamin D supplementation daily may be considered. Most prenatal vitamins contain $400 \mathrm{IU}$ of vitamin D. Perhaps concurrent with their glucose testing mid-pregnancy, women could have a serum or plasma $25(\mathrm{OH}) \mathrm{D}$ level measured on the same blood sample if the woman was found to have glucose intolerance. If the $25(\mathrm{OH}) \mathrm{D}$ level was found to be low, then women could initiate supplementation with 1,000-2,000 IU as suggested by ACOG. We believe that this targeted-testing and moderatedosing approach is reasonable until well-powered RCTs of larger doses of vitamin $\mathrm{D}$, or higher achieved $25(\mathrm{OH}) \mathrm{D}$ levels, are found to actually benefit the health of mothers with GDM or their infants. Ultimately, to make major advancements, we need to move beyond biomarkers and to conduct large multicentre RCTs that measure clinically important and relevant health outcomes for mothers with GDM and their infants.

Duality of interest The authors declare that there is no duality of interest associated with this manuscript.

Contribution statement Both authors were responsible for the conception and design of the manuscript, drafting the article and revising it critically for important intellectual content. Both authors approved the version to be published.

\section{References}

1. American College of Obstetricians and Gynecologists (2011) Committee opinion no. 504: screening and diagnosis of gestational diabetes mellitus. Obstet Gynecol 118:751-753

2. Jovanovic L, Pettitt DJ (2001) Gestational diabetes mellitus. JAMA 286:2516-2518

3. Reece EA, Leguizamon G, Wiznitzer A (2009) Gestational diabetes: the need for a common ground. Lancet 373:1789-1797
4. Committee on Practice Bulletins - Obstetrics (2013) Practice bulletin no. 137: gestational diabetes mellitus. Obstet Gynecol 122:406-416

5. Ferrara A, Kahn HS, Quesenberry CP, Riley C, Hedderson MM (2004) An increase in the incidence of gestational diabetes mellitus: Northern California, 1991-2000. Obstet Gynecol 103:526-533

6. Kjos SL, Buchanan TA (1999) Gestational diabetes mellitus. N Engl J Med 341:1749-1756

7. Vambergue A, Dognin C, Boulogne A, Rejou MC, Biausque S, Fontaine P (2008) Increasing incidence of abnormal glucose tolerance in women with prior abnormal glucose tolerance during pregnancy: DIAGEST 2 study. Diabet Med 25:58-64

8. Burris HH, Camargo CA Jr (2014) Vitamin D and gestational diabetes mellitus. Curr Diab Rep 14:451

9. Holick MF (2007) Vitamin D deficiency. N Engl J Med 357:266-281

10. Chu SY, Callaghan WM, Kim SY et al (2007) Maternal obesity and risk of gestational diabetes mellitus. Diabetes Care 30:2070-2076

11. Pittas AG, Lau J, Hu FB, Dawson-Hughes B (2007) The role of vitamin $\mathrm{D}$ and calcium in type 2 diabetes. A systematic review and meta-analysis. J Clin Endocrinol Metab 92:2017-2029

12. Wei SQ, Qi HP, Luo ZC, Fraser WD (2013) Maternal vitamin D status and adverse pregnancy outcomes: a systematic review and meta-analysis. J Matern Fetal Neonatal Med 26:889-899

13. Farrant HJ, Krishnaveni GV, Hill JC et al (2009) Vitamin D insufficiency is common in Indian mothers but is not associated with gestational diabetes or variation in newborn size. Eur J Clin Nutr 63:646-652

14. Burris HH, Rifas-Shiman SL, Kleinman K et al (2012) Vitamin D deficiency in pregnancy and gestational diabetes mellitus. Am J Obstet Gynecol 207(182):e181-e188

15. Cade C, Norman AW (1986) Vitamin $D_{3}$ improves impaired glucose tolerance and insulin secretion in the vitamin $\mathrm{D}$-deficient rat in vivo. Endocrinology 119:84-90

16. Bourlon PM, Billaudel B, Faure-Dussert A (1999) Influence of vitamin $\mathrm{D}_{3}$ deficiency and 1,25 dihydroxyvitamin $\mathrm{D}_{3}$ on de novo insulin biosynthesis in the islets of the rat endocrine pancreas. J Endocrinol 160:87-95

17. Rudnicki PM, Molsted-Pedersen L (1997) Effect of 1,25dihydroxycholecalciferol on glucose metabolism in gestational diabetes mellitus. Diabetologia 40:40-44

18. Soheilykhah S, Mojibian M, Moghadam MJ, Shojaoddiny-Ardekani A (2013) The effect of different doses of vitamin D supplementation on insulin resistance during pregnancy. Gynecol Endocrinol 29: 396-399

19. Asemi Z, Karamali M, Exmailzadeh A (2014) Effects of calciumvitamin D co-supplementation on glycaemic control, inflammation and oxidative stress in gestational diabetes: a randomised placebo-controlled trial. Diabetologia. doi:10.1007/s00125-0143293-x

20. Engelgau MM, Herman WH, Smith PJ, German RR, Aubert RE (1995) The epidemiology of diabetes and pregnancy in the U.S., 1988. Diabetes Care 18:1029-1033

21. Jelsma JG, van Poppel MN, Galjaard S et al (2013) DALI: vitamin D and lifestyle intervention for gestational diabetes mellitus (GDM) prevention: an European multicentre, randomised trial - study protocol. BMC Pregnancy Childbirth 13:142

22. American College of Obstetricians and Gynecologists (2011) ACOG Committee on Obstetric Practice. ACOG Committee opinion no. 495: vitamin D: screening and supplementation during pregnancy. Obstet Gynecol 118:197-198 